\title{
Vision System for 3D Reconstruction with Telecentric Lens
}

\author{
José Guadalupe Rico Espino ${ }^{1}$, José-Joel Gonzalez-Barbosa ${ }^{2}$, \\ Roberto Augusto Gómez Loenzo ${ }^{3}$, \\ Diana Margarita Córdova Esparza ${ }^{2}$, and Ricardo Gonzalez-Barbosa ${ }^{4}$ \\ 1 CIATEQ, A.C., Unidad Bernardo Quintana, México \\ 2 CICATA-IPN Unidad Querétaro, México \\ 3 Universidad Autónoma de Queretaro, México \\ 4 División Ciencias de la Vida, Universidad de Guanajuato, México
}

\begin{abstract}
This paper addresses 3D object reconstruction from images acquired by camera-telecentric lense array. Firstly, we present a geometric model of an array camera-telecentric lens. Then we developed and implemented the calibration process using a planar checkerboard pattern. At the same time, we developed a three-dimensional reconstruction system based on contour extraction on objects with dimensions less than $50 \mathrm{~mm}$ of diameter. Finally an analysis of the uncertainty model parameters and performance reconstruction of $3 \mathrm{D}$ objects are presented.
\end{abstract}

\section{Introduction}

In recent times, three-dimensional reconstruction of small objects has had major demand. To make this type of reconstructions, the distance between the optical system and the object must be small. One of the main problems with standard lenses is the perspective effect and lens distortion, at short distance, the object appears distorted. The telecentric lenses allow reducing some of the most common factors on the image acquisition: the magnification changes, due to the position of the optical system and the object, image distortion and perspective effects.

Three-dimensional reconstruction can be applied in several fields such as: manufacturing processes, metrology, robotics, biology, medicine, electronic microscopy, topography, computer-aided design, simulation, scientific visualization, just to name a few. Several methods have been proposed to determine the 3D surface profile. Traditionally, coordinate measurement machines (CMMs) have been used for surface profile measurement. While it is still extensively used in industries, however there are limitations such as low speed and high cost. In metrology applications it requires fast and soft material measurement. In [1] is described a research project T'nD (Touch and Design), which is a free-form modeling system with another articulated arm that has two 3-DOF hap tic devices connected to a ruler tool by means of spherical joints. This design allows the scraping of a digital surface by sweeping motions with the ruler. Handheld tools without force feedbacks have also been used for $3 \mathrm{D}$ digital form control. Optical 
methods, such as interferometry [4, stereo vision [9], 2, structured light projection [6], 3] and shape from focus/defocus [7], [12], have long received extensive attention for 3D objects reconstruction. Unlike CMM, measurement systems developed from optical principles are non-contact in nature, this way it prevents the mechanical wear and surface deformation problem. One of the main features of these methods is its high resolution and large measurement range, measurement with millions of points can be accomplished within a few seconds. In [2] describes the development of a novel volumetric method for small objects. The authors use a binocular machine vision system composed of camera-telecentric lense. The authors present a camera model for telecentric lenses, an algorithm for calibration and 3D reconstruction. The calibration algorithm calculates the parameters incrementally. First the camera model parameters are computed, using these values distortion parameters are calculated and finally, all the parameters values are refined by iterative optimization algorithm. The $3 \mathrm{D}$ reconstruction is developed by triangulation between shapes detected on two images by correspondence. In [9] is presented a shape representation for objects based on 3D contour fragments that build a 3D Contour Cloud. The approach for the automatic reconstruction of such 3D Contour Clouds from calibrated stereo image sequences includes a novel idea on the reconstruction of $3 \mathrm{D}$ contour fragments from stereo frame pairs and a robust Structure and Motion analysis. The stereo reconstruction is based in shape correspondences, the search space is limited to a subset of contours by only taking into account those contour fragments which lie in regions restricted by the epipolar lines. In [8], a combination of surface fitting and registration based on a squared distance minimization algorithm is discussed and applied to a constrained reverse engineering of CAD models

In this work is considered a system of the second classification, which has a digital camera and telecentric lens to acquire images to be used in threedimensional reconstruction of the object. The optical system formed by a camera and a perspective lens is the most common, so this model and calibration have been extensively studied. However, the system with camera and telecentric lens is a model with less studies,we must develop and implement the calibration model that allows three-dimensional reconstruction of pieces with less than $50 \mathrm{~mm}$ of diameter.

\section{Experimental Development}

In order to perform measurement, a mathematical relationship between the three-dimensional measurement and the coordinates array camera-telecentric lense must be established. To accomplish this, a precise system model for array camera-telecentric lense pair is developed. A calibration process is subsequently performed to obtain parameters of the proposed system model.

\subsection{Geometrical Model Approach}

The orthographic geometry, as illustrated in Figure 1 is the projection of a threedimensional entity on a plane image by a set of parallel rays orthogonal to this 
plane. In Figure 1, we have that $x=X$, and $y=Y$. Where $(X, Y)$ and $(x, y)$ denote the coordinates of the object and image respectively.

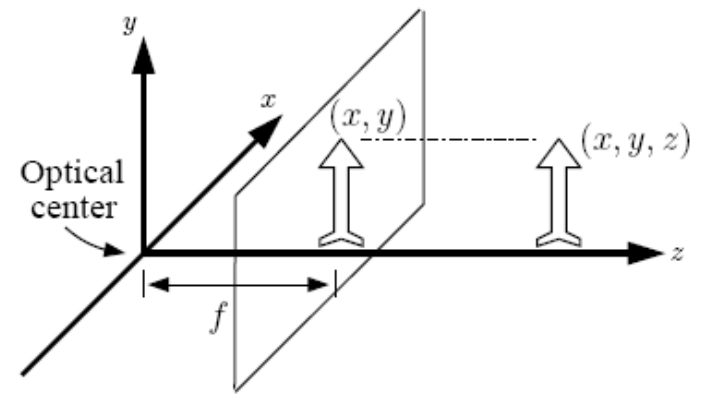

Fig. 1. Orthographic projection model of a real object onto the image plane

The orthographic projection $\mathbf{q}=[x, y, 1]^{T}$ of a point $\mathbf{Q}=[X, Y, Z, 1]^{T}$ defined in world coordinates can be modeled by equation 1 .

$$
\mathbf{q}=\mathrm{KEQ}
$$

$\mathbf{K}$ is defined as the intrinsic parameter matrix and $\mathbf{E}$ as the extrinsic parameters matrix, defined by equation 2 .

$$
\mathbf{K}=\left[\begin{array}{cccc}
\alpha & \gamma & 0 & 0 \\
0 & \beta & 0 & 0 \\
0 & 0 & 0 & 0
\end{array}\right] ; \mathbf{E}=\left[\begin{array}{cc}
\mathbf{R} & \mathbf{T} \\
\mathbf{0} & 1
\end{array}\right]
$$

The elements of $\mathbf{K}$ includes

$\alpha$ and $\beta$ the scale factors in image $x$ and $y$ axes, and $\gamma$ the parameter describing the skewness of the two image axes. The matrix $\mathbf{E}$ represents the rigid transformation that carried out the world coordinate system to the imagecoordinate system, composite of a rotation $\mathbf{R} \in \Re^{3 x 3}$ and a translation $\mathbf{T} \in \Re^{3}$.

\subsection{Estimation of Intrinsic and Extrinsic Parameters}

This section describes a general method for estimating model parameters of the above section. In [5] is presented a model for estimating the intrinsic and extrinsic parameters separately, using multiple images of standard planar checkerboard pattern. The procedure for estimating the intrinsic and extrinsic parameters is developed based on this model. The method assumes that the calibration pattern is a plane on $Z=0$, thus eliminating the third row and third column in E (equation 2), obtaining:

$$
\mathbf{q}=\mathbf{K}_{c} \mathbf{E}_{c} \mathbf{Q}_{c}=\left[\begin{array}{lll}
\alpha & \gamma & 0 \\
0 & \beta & 0 \\
0 & 0 & 1
\end{array}\right]\left[\begin{array}{ccc}
r_{11} & r_{12} & t_{1} \\
r_{21} & r_{22} & t_{2} \\
0 & 0 & 1
\end{array}\right]\left[\begin{array}{c}
X \\
Y \\
1
\end{array}\right]
$$


Where $\mathbf{R}_{c} \in \Re^{2 \times 2}$ and $\mathbf{T}_{c} \in \Re^{2}$ represent the truncated matrices for rotation and translation, respectively.

The estimation of the intrinsic parameters are calculated from the definition of the homography $\mathbf{H} \in \Re^{3 x 3}$ which relates features of a calibration pattern (e.g. coordinates in the corner of a checkerboard type pattern) with the image.

So, this way $\mathbf{q}=\mathbf{H Q}_{c}$ that substituting in equation 3 and solving for $\mathbf{H}$, we obtain equation 4 .

$$
\mathbf{H}=\mathbf{K}_{c} \mathbf{E}_{c}
$$

Now, multiplying both sides of the equation $4, \mathbf{K}_{c}^{-T}$ and subsequently by their respective transposed is obtained:

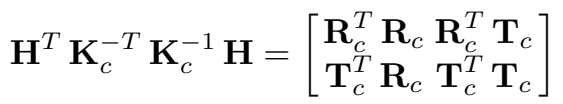

Equation 5 can be used to resolve intrinsic parameters, in particular using the upper left submatrix $\mathbf{R}_{c}^{T} \mathbf{R}_{c}$. First, the rotation matrix $\mathbf{R}$ from equation 2 is expressed in terms of $\mathbf{R}_{c}$ :

$$
\begin{gathered}
\mathbf{R}=\left[\begin{array}{cc}
\mathbf{R}_{c} & \mathbf{B} \\
\mathbf{D}^{T} & d
\end{array}\right] \\
\mathbf{R}_{c}^{T} \mathbf{R}_{c}+\mathbf{D ~}^{T}=\mathbf{I}_{2} \\
\mathbf{D}^{T}=\left[\begin{array}{l}
d_{11} \\
d_{21}
\end{array}\right]\left[\begin{array}{ll}
d_{11} & d_{21}
\end{array}\right]=\left[\begin{array}{cc}
d_{11}^{2} & d_{11} d_{21} \\
d_{11} d_{21} & d_{21}^{2}
\end{array}\right] \\
\operatorname{det}\left(\mathbf{D} \mathbf{D}^{T}\right)=d_{11}^{2} d_{21}^{2}-d_{11} d_{21} d_{11} d_{21}=0
\end{gathered}
$$

The equation 7 can be defined as follows:

$$
\operatorname{det}\left(\mathbf{R}_{c}^{T} \mathbf{R}_{c}-\mathbf{I}_{2}\right)=0
$$

Developing equations 5 and 8 is obtained:

$$
\begin{array}{r}
k_{1}-k_{2}\left(h_{21}^{2}+h_{22}^{2}\right)-k_{3}\left(h_{11}^{2}+h_{12}^{2}\right)+2 k_{4}\left(h_{11} h_{21}+h_{12} h_{22}\right)= \\
-\left(h_{12} h_{21}-h_{11} h_{22}\right)^{2}
\end{array}
$$

Now then, there will be a homography for each image of the checkerboard pattern, which is defined by equation 10 .

$$
\mathbf{H}_{c}=\left[\begin{array}{lll}
h_{11} & h_{12} & h_{13} \\
h_{21} & h_{22} & h_{23} \\
h_{13} & h_{32} & h_{33}
\end{array}\right]
$$

where, $h_{31}=0, h_{32}=0$ and $h_{33}=1$. So then, requires a minimum of 4 images (the calibration pattern) to obtain $k_{1}, k_{2}, k_{3}$ and $k_{4}$, and obtain the intrinsic 
parameters: $\alpha, \beta$ and $\gamma$. Finally, the extrinsic parameters $\mathbf{T}_{c}$ and $\mathbf{R}_{c}$ can be found from equation 4. The method for calculating these parameters is described in the next section.

\subsection{Calibration Method}

A standard method to calculate intrinsic and extrinsic parameters of a vision system is to obtain 3D images of an object known and find the set of parameters. The set of parameters found will project the observed points from 3D world coordinates and the coordinates of the corresponding pixel in the calibration image better. To develop the calibration process, we use a plane calibration pattern of the checkerboard type. The calibration pattern brand is Opto-Engineering model PT036-056

The process that relates the points pattern with the image points is described in Section 2.1] specifically in equation 11. Then, the set of process parameters are unknown, and these are : (1) scaling factors $(\alpha, \beta),(2)$ skew factor $(\gamma)$ and factors of spatial position $\left(R_{c}, T_{c}\right)$.

The calibration problem is to find a total of 9 scalars describing the projection of world coordinates in $3 \mathrm{D}$ to the image coordinate. The procedure to obtain these values is developed in 5 stages, which are described below:

Stage 1: Acquisition of images: capture and storage of the 4 calibration images. In Figure 2 (a), is observed one picture that were used to calibrate the system. The images are in gray scale with a resolution of 1280 x 960 pixels.

Stage 2: Extraction of the intersections of black and white cadres of the calibration pattern. The coordinates obtained correspond to the positions they have in the checkerboard pattern, taking the corner as the origin and from which are measured $1.35 \mathrm{~mm}$ increments, to obtain the coordinates in millimeters, so the coordinates of the intersections represent the position in pixels, in the image. Figure 2 (b) shows a detail of the intersections (corners), found in one of the images of the calibration.

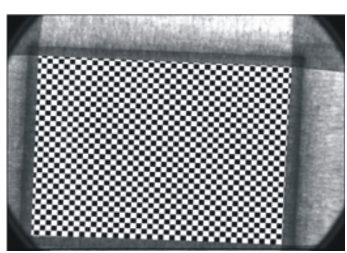

(a)
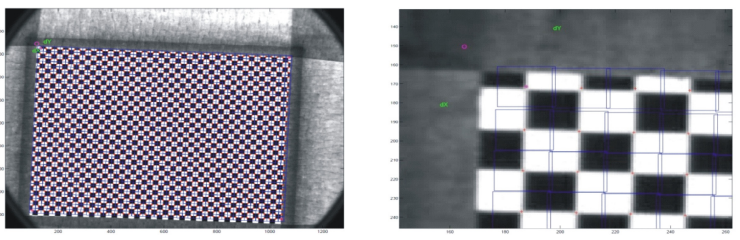

(b)

Fig. 2. (a) Calibration pattern images in grayscale (b) Corners found in the left image of the calibration, the blue boxes indicate the search area of the corner and the red cross in the corner found

Stage 3: Compute the homographies for each image, using equation 10, where the terms $h_{11}, h_{12}, h_{13}, h_{21}, h_{22}$ and $h_{23}$ are arranged in a vector in order to establish the system of equations as follow: 


$$
\mathbf{H}_{C A L}=\left[\begin{array}{cccccc}
x_{1} & y_{1} & 1 & 0 & 0 & 0 \\
0 & 0 & 0 & x_{1} & y_{1} & 1 \\
\vdots & \vdots & \vdots & \vdots & \vdots & \vdots \\
x_{j} & y_{j} & 1 & 0 & 0 & 0 \\
0 & 0 & 0 & x_{j} & y_{j} & 1
\end{array}\right]\left[\begin{array}{c}
h_{11} \\
h_{12} \\
h_{13} \\
h_{21} \\
h_{22} \\
h_{23}
\end{array}\right]=\left[\begin{array}{c}
u_{1} \\
v_{1} \\
\vdots \\
u_{j} \\
v_{j}
\end{array}\right]
$$

The value of $j$ is the total number of points found in the corner extraction step. $x_{j}$ and $y_{j}$, indicate the coordinates of the points of the world, $u_{j}$, and $v_{j}$ indicate the coordinates of the points in the image

Stage 4: Intrinsic parameters: the values of $h_{11}, h_{12}, h_{13}, h_{21}, h_{22}$ and $h_{23}$ solve the system of equations $C k=D$, which developed in matrix form is denoted by equation 12, for each image obtained.

$$
\left[\begin{array}{llll}
C_{1} & C_{2} & C_{3} & C_{4}
\end{array}\right]^{T}\left[\begin{array}{llll}
k_{1} & k_{2} & k_{3} & k_{4}
\end{array}\right]^{T}=\left[\begin{array}{llll}
D_{1} & D_{2} & D_{3} & D_{4}
\end{array}\right]^{T}
$$

Now $C_{1}, C_{2}, C_{3}$ and $C_{4}$ are defined by: $1-\left(h_{21}^{2}+h_{22}^{2}\right)-\left(h_{11}^{2}+h_{12}^{2}\right)+2\left(h_{11} h_{21}+\right.$ $\left.h_{12} h_{22}\right)$, where $h_{11}, h_{12}, h_{21}$ and $h_{22}$ correspond to the value of each homography obtained above (remember that there is one for each image of the calibration). Moreover $D_{1}, D_{2}, D_{3}$ y $D_{4}$, are defined by: $-\left(h_{12} h_{21}-h_{11} h_{22}\right)^{2}$. These definitions correspond to equation 9 . The vector defined by $k_{1}, k_{2}, k_{3}$ and $k_{4}$ represents the necessary scalars to obtain the values of the intrinsic parameters of the camera

Thus, the values of camera intrinsic parameters are defined using the equations defined in 12, which allow the values of $\alpha \beta$ and $\gamma$, scale factors and skew factor, respectively. So then, the equations that are required to obtain the above values are defined by equation 13 .

$$
\alpha=\sqrt{\frac{k_{2} k_{3}-k_{4}^{2}}{k_{3}}}, \beta=\sqrt{k_{3}}, \gamma=\sqrt{\frac{k_{2} k_{3}-k_{1}}{k_{3}}}
$$

Stage 5: Extrinsic Parameters require the values of homographies previously obtained: $h_{11}, h_{12}, h_{13}, h_{21}, h_{22}$ and $h_{23}$ complementing $\mathbf{H}_{C A L}$ homography matrix from equation 10. Now, as $\mathbf{q}=\mathbf{K}_{c} \mathbf{E}_{c} \mathbf{Q}_{c}$, and $\mathbf{q}=\mathbf{H}_{c} \mathbf{Q}_{c}$ can be substituted to sort out $\mathbf{E}_{c}$ to obtain the extrinsic parameters of the camera, by applying equation 14, resulting

$$
\mathbf{E}_{c}=\mathbf{K}_{c}^{-1} \mathbf{H}_{c}
$$

And finally, solving the above equation and substituting the values of the obtained homographies for each image of the calibration, we obtain the equation 15 that allows to know the spatial orientation of the object as viewed from the camera.

$$
\mathbf{E}_{c}=\left[\begin{array}{ccc}
-0.0441 & 0.9934 & 9.6526 \\
0.9986 & 0.0410 & 12.5414 \\
0 & 0 & 1
\end{array}\right]
$$




\section{Numerical Validation}

To validate the calibration, is used as a reference (in world coordinates), a calibrated previously digital caliper brand: Mitutoyo, Model: 500-196-20, with a range from 0 to $150 \mathrm{~mm}$ with an accuracy of $0.01 \mathrm{~mm}$. The procedure for validation of calibration is based on computing the distance between the contour points of the digital caliper used for measuring from images.

\section{Results}

This section describes the experiments conducted to evaluate the calibration and implementation of the different components of the proposed 3D reconstruction system. It is spat into three sections, each addressing a possible implementation and the means to evaluate the effectiveness of different constituents of the present system

\subsection{Uncertainty of Intrinsic Parameters}

The uncertainty of the calibration parameters is calculated by Monte Carlo. In [10] is used a computer-generated random numbers to simulate many synthetic data sets. The uncertainty values reported are in range $1 \sigma$, which corresponds to a confidence level of $68 \%$. The Uncertainty values of the intrinsic parameters are given in the Table 1

Table 1. Results on the measurement of the intrinsic parameters and uncertainties obtained

\begin{tabular}{|c|c|c|}
\hline Parameters & Measurement values & Uncertainty \\
\hline$\alpha$ & 15.9029 & 0.030091763 \\
\hline$\beta$ & 15.8597 & 0.000566170 \\
\hline$\gamma$ & 0.0446 & 0.009720238 \\
\hline$r_{11}$ & -0.0413 & 0.000553120 \\
\hline$r_{12}$ & 0.9996 & 0.001907245 \\
\hline$r_{21}$ & 1.0048 & 0.000044290 \\
\hline$r_{22}$ & 0.0444 & 0.000023499 \\
\hline$t_{1}$ & -12.1379 & 0.024776660 \\
\hline$t_{2}$ & -10.2556 & 0.001122942 \\
\hline
\end{tabular}

\subsection{Validation of the Calibration}

To validate the calibration, is used as a reference a digital caliper with a range from 0 to $150 \mathrm{~mm}$ with an accuracy of $0.01 \mathrm{~mm}$. The procedure for validation of calibration is based on measuring the distance between two parallel lines. The opening of the digital caliper rule. The processing of these images is done by a sub pixel edge detection algorithm, and an algorithm to determine the distance 
(in millimeters) between points of the digital caliper image that belongs to the lines. In total, there were 6 measurements at various distances: 5, 10, 15, 20, 25 and $30 \mathrm{~mm}$, with 3 repetitions each one, for a total of 18 readings. Table 2 shows the result of measuring different digital caliper openings. The Figure 3 shows the distance measurement for the three images for each measure.
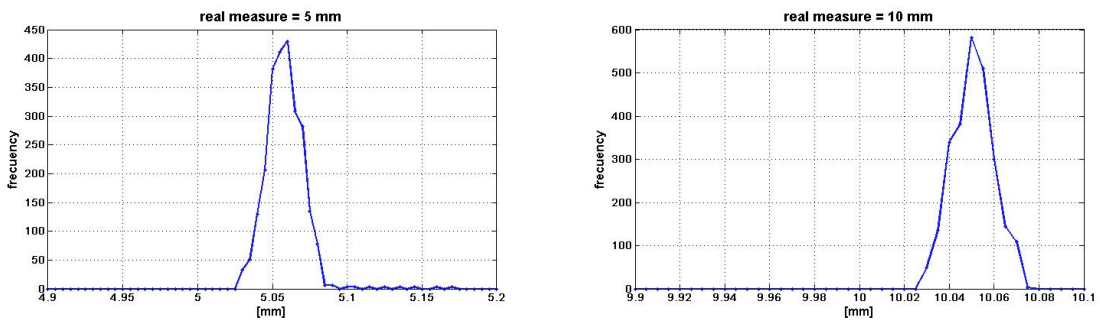

Fig. 3. Histograms of the distances calculated for the digital caliper with an opening 5, 10 millimeters. The histograms are calculated using three images for each measure.

Table 2. Different values of the digital caliper openings

\begin{tabular}{|c|c|c|}
\hline Real value $(\mathrm{mm})$ & Averange $(\mathrm{mm})$ & Standard deviation $(\mathrm{mm})$ \\
\hline 5 & 5.0585 & 0.0133 \\
10 & 10.0507 & 0.0091 \\
15 & 15.0467 & 0.0103 \\
20 & 20.0351 & 0.0106 \\
25 & 25.0228 & 0.0134 \\
30 & 30.0087 & 0.0118 \\
\hline
\end{tabular}

In this stage has been validated to quantify the size of opening, the terminal of a digital caliper, obtained by the camera-telecentric lens system, in order to quantify the tridimensional reconstructor based on camera- telecentric lense. The achieved precision in [2] is high, providing a standard deviation of $0.04 \mathrm{~mm}$.

\subsection{Three Dimensional Reconstruction}

In the case of finished products is a key point to know and verify the quality with which it is prepared to comply with the requirements relating to its development. So then, knowing the final product characteristics and quality tests, has to focus on products in the commercial sector to provide a tool to take some of these tests. In particular, it provides the dimensions of the piece and it is developed the three-dimensional reconstruction. In this case is reconstructed a section of an object, using an algorithm that can recover the shape of the object and it is aligned on an axis of rotation as [1]. It is therefore necessary to know the ratio between the world in millimeters and the world in pixels. This transformation can be know clearing the equation 1, the value of $Q_{c}$, to obtain equation 16 . 


$$
\mathbf{Q}_{c}=\mathbf{E}_{c}^{-1} \mathbf{K}_{c}^{-1} \mathbf{q}
$$

where

$$
\mathbf{E}_{c}^{-1} \mathbf{K}_{c}^{-1}=\left[\begin{array}{ccc}
-0.0413 & 0.9996 & -12.1379 \\
1.0048 & 0.0444 & -10.2556 \\
0 & 0 & 1
\end{array}\right]\left[\begin{array}{ccc}
0.0629 & -0.0002 & 0 \\
0 & 0.0631 & 0 \\
0 & 0 & 1
\end{array}\right]
$$

Where $\mathbf{Q}_{c}$ corresponds to the coordinates in millimeters and $\mathbf{q}$ pixels. $\mathbf{E}_{c}$ and $\mathbf{K}_{c}$ matrices are calculated in Section 2.2. So then, in Figure 4(a) shows the alignment of the silhouettes on a rotation axis, with dimensions in millimeters (area of approximately $50 \mathrm{~mm}$ by $45 \mathrm{~mm}$ ). Also, it shows the process of reconstruction of the object with different silhouettes. Figure 4 (b) presents the results of the final reconstruction of textured object.

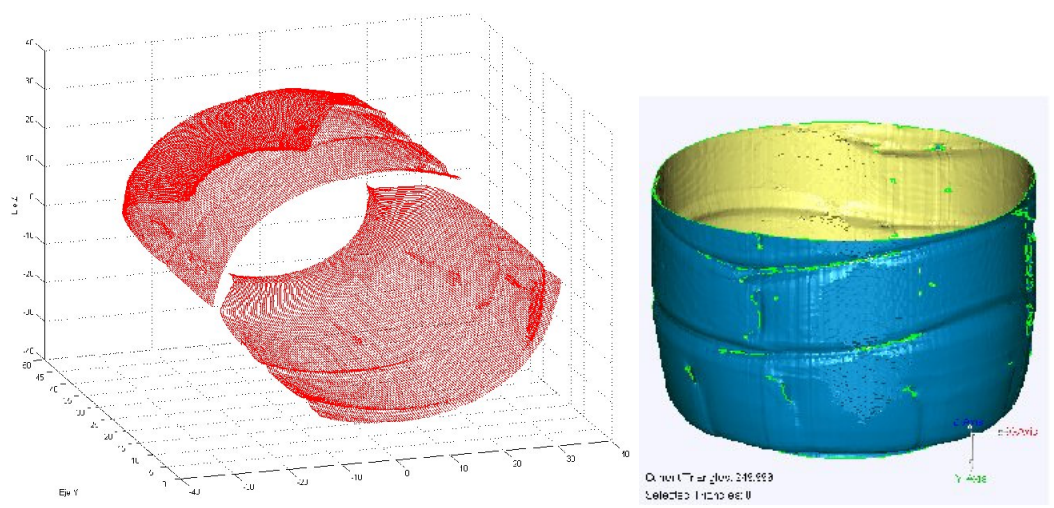

Fig. 4. Left: Three-dimensional reconstruction with 120 silhouettes. Right: Threedimensional reconstruction with texture of the plastic bottle segment.

\section{Conclusions and Futur Works}

A geometric model of an array camera-telecentric lens has been presented, it allows relating the world coordinates (in millimeters) with the coordinates of the image obtained by the camera (in pixels). It also allows finding the dimensional real values from the images. The proposed method achieves the sizing of objects with measures less than $50 \mathrm{~mm}$ of diameter. This leads to the quantification of the dimensions of a plastic bottle for water storage, which in turn allows the three-dimensional reconstruction using a reconstruction algorithm by means of the object silhouettes.

Future work is directed to quantify the reconstruction error using more accurately patterns. The calibration and reconstruction process has been developed using Matlab, but our efforts are aimed at developing algorithms in $\mathrm{C}++$. Future 
work is directed to quantify the reconstruction error using patterns more accurately. The future prototype will be incorporated to a structured light pattern projector in order to reconstruct concaves zones that can't be reconstructed.

Acknowledgements. This work was supported partially by CONACYT grant CB2005-01-51004/25293 and SIP-IPN grant 20120472.

\section{References}

1. Bordegoni, M., Cugini, U.: Create free-form digital shapes with hands. In: Proceedings of the 3rd International Conference on Computer Graphics and Interactive Techniques in Australasia and South East Asia, GRAPHITE 2005, pp. 429-432. ACM, New York (2005)

2. Gorpas, D., Politopoulos, K., Yova, D.: A binocular machine vision system for three-dimensional surface measurement of small objects. Comp. Med. Imag. and Graph. 31(8), 625-637 (2007)

3. Guerra, E., Villalobos, J.: A three-dimensional automated visual inspection sustem for smt assembly. Computers and Industrial Engineering 40, 175-190 (2001)

4. Kong, B., Kim, S.: Portable inspection of precision surface by phase shifting interferometry with automatic suppression of phase shift errors. Optical Engineering 34, 1400-1404 (1995)

5. Lanman, D., Cabrini, H.D., Taubin, G.: Shape from depth discontinuities under orthographic projection. Technical Report, Division of Engineering (2009)

6. Lee, M., De Silva, C., Croft, E., Wu, Q.: Machine vision system for curved surface inspection. Machine Vision and Applications 12, 177-188 (2000)

7. Leopold, J., Gunther, H., Leopold, R.: New developments in fast 3d-surface quality control. Measurement 33, 179-187 (2003)

8. Liu, Y., Pottmann, H., Wang, W.: Constrained 3d shape reconstruction using a combination of surface fitting and registration. Comput. Aided Des. 38, 572-583 (2006)

9. Potsch, K., Pinz, A.: 3d geometric shape modeling by 3d contour cloud reconstruction from stereo videos. In: Wendel, A., Sternig, S., Godec, M. (eds.) Proceedings of the Computer Vision Winter Workshop, CVWW 2004, pp. 429-436 (2011)

10. Press, W.H., Teukolsky, S.A., Vetterling, W.T., Flannery, B.P.: Numerical recipes in $\mathrm{C}$ (2nd ed.): the art of scientific computing. Cambridge University Press, New York (1992)

11. Taubin, G., Crispell, D., Lanman, D., Sibley, P., Zhao, Y.: Shape from Depth Discontinuities. In: Nielsen, F. (ed.) ETVC 2008. LNCS, vol. 5416, pp. 216-237. Springer, Heidelberg (2009)

12. Toyoura, M., Iiyama, M., Funatomi, T., Kakusho, K., Minoh, M.: 3d shape reconstruction from incomplete silhouettes in multiple frames. In: ICPR, pp. 1-4 (2008) 